CIRJE-F-1053

\title{
Robust Technical Trading with Fuzzy Knowledge-based Systems
}

\author{
Masafumi Nakano \\ Graduate School of Economics, The University of Tokyo \\ Akihiko Takahashi \\ The University of Tokyo \\ Soichiro Takahashi \\ Graduate School of Economics, The University of Tokyo
}

July 2017

CIRJE Discussion Papers can be downloaded without charge from:

http://www.cirje.e.u-tokyo.ac.jp/research/03research02dp.html

Discussion Papers are a series of manuscripts in their draft form. They are not intended for circulation or distribution except as indicated by the author. For that reason Discussion Papers may not be reproduced or distributed without the written consent of the author. 


\title{
Robust technical trading with fuzzy knowledge-based systems *
}

\author{
Masafumi Nakano, Akihiko Takahashi, Soichiro Takahashi \\ Graduate School of Economics, University of Tokyo, \\ 7-3-1 Hongo Bunkyo-ku, Tokyo, Japan, 113-0033
}

July 2, 2017

\begin{abstract}
This paper proposes a framework of robust technical trading with fuzzy knowledge-based systems (KBSs). Particularly, our framework consists of two modules, i.e., (i) a module for preparing candidate investment proposals and (ii) a module for their evaluation to construct a well-performed portfolio.

Moreover, our framework effectively utilizes fuzzy KBSs for representation of human expert knowledge: Precisely, in the 1st module, three sets of fuzzy IF-THEN rules implement linguistic technical trading rules, which are designed specifically for getting well performance in different market phases. On the other hand, the 2nd module exploits fuzzy logic to evaluate the prepared investment candidates in terms of multilateral performance measures frequently used in practice.

In an out-of-sample numerical experiment, our framework successfully generates a series of portfolios, which show long-term satisfactory records in the prolonged slumping Japanese stock market.
\end{abstract}

Keywords: knowledge-based system, fuzzy logic, technical trading, market phase, Japanese stock market

\footnotetext{
*forthcoming in "Frontiers in Artificial Intelligence and Applications"
} 


\section{Introduction}

It is well-recognized both in academia and industry that financial markets are highly complex and non-linear systems with large noise, which are affected by economic, political, geopolitical and psychological factors. Therefore, for decision making in the financial markets, soft computing techniques are effectively used to deal with non-linearity and uncertainty (e.g., [1]).

Fuzzy logic, initiated by Zadeh [2], is one of the soft computing techniques, which is applied with great success in financial investment problems. Especially, the concept of fuzzy logic is utilized in portfolio optimization problems because it enables to represent imperfect knowledge or ambiguity for the future asset return. For example, although a mean-variance (MV) portfolio [3] has been one of the most famous strategies, there is a well-known serious problem that the direct MV optimization amplifies the effects of estimation errors (e.g., [4]). Consequently, many researchers introduce the fuzziness in portfolio optimization problems from various perspectives (e.g., $[5-22])$.

On the other hand, fuzzy systems (e.g., $[23,24]$ ) are effective to construct knowledge-based systems (KBSs) or expert systems (ESs) for trading strategies with technical and fundamental analysis (e.g., [25-32] ). In trading practice, domain expert knowledge is often expressed in a linguistic form, which can be incorporated into trading strategies through IF-THEN rules of fuzzy systems.

This paper proposes a framework for robustly performing technical trading by making use of fuzzy KBSs. In particular, our framework consists of two modules; (i) a candidate preparation module and (ii) a performance evaluation module. More precisely, we first prepare various investment candidates in the 1st module based on three types of fuzzy rule bases for technical trading. Then, the 2nd module evaluates historical records of the investment candidates to generate a promising portfolio. Here, we use a variety of performance measures, which are practically well-known to investment experts such as hedge fund managers.

The motivation of our work is how to find out the effective investment proposals from numerous possible options under restricted prior knowledge. Especially, for robustly performed technical trading, it is important to properly judge whether the market is in a trend or range phase, though it is quite difficult in general. Then, in the 1st module, we prepare the investment candidates based on several patterns of the trading rules, which are specialized to perform well in different market phases. As a result, since fuzzy inference of the 2 nd module employs the candidates showing high records in terms of multilateral criteria, our framework is robust against the misidentification of market phases, in principle. Importantly, as performance measures are the most critical and essential investment objectives for investors, our framework is also expected to directly link to high performance.

To show the validity, we implement an out-of-sample numerical experiment for Japanese stock market suffering from long-term slump. Besides, we use various performance criteria, i.e., compound return (CR), standard deviation (SD), downside deviation (DD), maximum drawdown (MDD), Sharpe ratio (ShR), Sortino ratio (SoR) and Stering ratio (StR).

The remainder of this paper is organized as follows. Section 2 summarizes related works. Section 3 presents our framework architecture: fuzzy logic-based technical trading and performance evaluation. Section 4 shows the results of out-of-sample numerical experiments. Finally, Section 5 concludes.

\section{Related works}

In this section, we shortly review previous works for application of fuzzy set theory to three topics of finance, that is, time-series prediction, technical/fundamental trading and modern portfolio 
theory.

\subsection{Application to financial time-series prediction}

Fuzzy logic is frequently used to develop KBSs for financial time-series prediction due to its general applicability. For example, Korol [37] builds a fuzzy system for forecasting exchange rates based on various economic factors such as GDP and inflation, which achieves lower mean absolute percentage error than other statistical models and artificial neural network approaches.

Cai, Zhang, Zheng, and Leung [38] develop a new fuzzy time series forecasting model. Particularly, they exploit ant colony optimization to promote the forecasting performance. Further, the auto-regression method is adopted to make better use of historical information. The new model combined with these techniques is shown to be more effective than existing models through the application to Taiwan capitalization weighted stock index.

Hadavandi, Shavandi, and Ghanbari [39] construct a stock price forecasting expert system based on genetic fuzzy systems and artificial neural networks. More precisely, after step-wise regression analysis determines factors having most influence on stock prices, they divide raw data into multiple clusters by self-organizing map neural network. Then, each cluster is fed into genetic fuzzy systems with the ability of rule base extraction and database tuning.

Singh and Borah [40] develop a new high-order fuzzy time-series model, where artificial neural network based architecture is exploited for defuzzification. In particular, they discuss the importance on "lengths of intervals" for time-series and introduce a repartitioning discretization approach. Their methodology is validated with daily temperature data and stock price data.

\subsection{Application to technical/fundamental trading}

Since fuzzy IF-THEN rules are helpful to quantitatively express expert knowledge for technical and fundamental trading, various researchers have applied fuzzy systems to this field. As a pioneering work, in 1991, Kosaka, Mizuno, Sasaki, Someya, and Hamada [27] propose a framework for fuzzy rule-based technical trading, which is illustrated by single stock data.

Simutis [30] develops a computer software for fuzzy logic-based stock trading with evolutionary programming methods, where technical and fundamental information is exploited to produce buy/sell signals. The system with datasets of US stock markets shows high performance over two year investment period (1996-1998).

Lam [28] proposes a fuzzy expert system with genetic algorithm for stock trading, where inputs are twelve technical indicators and outputs are buy/sell signals. The numerical test with Hong-Kong stock market data illustrates that the system performs better than buy-and-hold strategies while individual technical indicator seems unreliable itself.

Dourra and Siy [25] present a trading system using technical analysis and fuzzy logic, which features a convergence module mapping technical indicators into new inputs for a Mamdani type fuzzy system. Their trading system is applied to stock price data of four companies with investment strategies based on two types of buy/sell trigger identification, which outperforms buy-and-hold of S\&P 500.

Dymova, Sevastianov, and Bartosiewicz [26] integrate fuzzy logic and methods of the DempsterShafer theory (the so-called rule-base evidential reasoning) to build expert systems for investment with technical indicators as inputs and buy/hold/sell signals as outputs. The system is tested by a Warsaw stock index futures contract and performs well.

Lincy and John [29] construct a fuzzy inference system for daily stock trading, in which inputs are mean and standard deviation of historical returns as well as earnings per share (EPS) while outputs are buy, hold and sell signals. Their experiment based on dataset of 25 stocks in 
NASDAQ stock exchange shows that the ES performs better than simple application of famous technical indicators and several existing models.

In Yunusoglu and Selim [31], the proposed ES provides an optimal portfolio in terms of rating point which is created by integrating technical and fundamental evaluation based on various fuzzy rules. The system incorporates investor's risk preference through the predetermined weight constraints. Using the data of 61 stocks in Istanbul Stock Exchange National-100 Index during 2002-2010, they demonstrate its validity by comparison with the benchmark index.

Chourmouziadis and Chatzoglou [32] design a short-term trading ES which tells how much amount should be invested in a risky asset based on fuzzy rules. One of its important features is the use of rare technical indicators, i.e. Parabolic SAR and GANN-HiLo. In the numerical example with the daily data of Athens Stock Exchange General Index over a period of more than 15 years, the strategy with their ES is shown to be superior to the buy-and-hold strategy.

\subsection{Extension of Markowitz model with fuzzy logic}

The concept of fuzziness including vagueness of human reasoning is also applied to the modern portfolio theory, introduced by Markowitz [3]. As a pioneering work, in 1997, Watada [5] introduces vague targets for portfolio expected return and risk in the Markowitz model. As well, in 1998, Ramaswamy [6] introduces fuzzy decision theory (Bellman and Zadeh [41]) into portfolio selection to represent the situation that an investor allows target rate of return is not necessarily attained if his/her market scenario turns out to be incorrect. Moreover, Fang, Lai, and Wang [9] consider a fuzzy portfolio rebalancing model with transaction costs, where the required levels of portfolio liquidity in addition to return and risk are regarded as fuzzy numbers.

At the same time, asset returns are also regarded as fuzzy numbers in portfolio selection problems. For instance, Tanaka, Guo, and Türksen [7] assume that returns of securities follow possibility distributions (Zadeh [42]) in a framework of mean-variance analysis.

In these formulation, various risk measures are studied and advanced to express different investors' risk preferences which is not necessarily represented by existing approaches. Huang [10] proposes a fuzzy portfolio selection model using a new definition of investment risk based on credibility theory, which is solved by genetic algorithm. Also, Liu [15] considers a fuzzy portfolio optimization problem with mean-absolute deviation function and Zadeh's extension principle, where two level mathematical programs are transformed into a pair of ordinary one-level linear ones. Likewise, Zhou, Li, and Pedrycz [22] introduce a concept of fuzzy semientropy to quantify the down side uncertainty, which formulates two mean-semi-entropy portfolio selection models with simulation-based genetic algorithm. Moreover, Nguyen, Gordon-Brown, Khosravi, Creighton, and Nahavandi [19] introduce a new portfolio risk measure and fuzzy Sharpe ratio. Correspondingly, two portfolio optimization problems are formulated and solved by fuzzy approach or genetic algorithm, where an experimental result shows their approach is more effective than the existing one.

Since the higher moment information of asset return gets more important, many researchers also incorporate it into the fuzzy framework. For example, Wang, Wang, and Watada [21] develop a VaR-based fuzzy portfolio selection model, which is solved by an improved particle swarm optimization algorithm. Also, Nguyen and Gordon-Brown [18] apply constrained fuzzy analytic hierarchy process methods to incorporate higher moment information such as skewness and kurtosis as well as volatility into portfolio selection. Further, Li, Guo, and Yu [12] propose a new mean-variance-skewness fuzzy portfolio model, which is shown to be more diversified than an existing credibilistic model.

In turn, for better portfolio performances, it seems important to employ different kinds of criteria including qualitative ones. From this perspective, $\mathrm{Li}$ and $\mathrm{Xu}$ [14] address a multi-objective fuzzy portfolio selection model with genetic algorithm, where investors take into consideration 
various elements including historical price data, their own investment attitudes and experts' opinions in addition to return, risk and liquidity. As well, Mehlawat [16] presents a crediblistic mean-entropy model for multi-objective multi-period portfolio selection, whose major criteria are wealth, risk, transaction cost, liquidity, and number of investment assets. Besides, Pai [20] discusses a metaheuristic portfolio optimization with multiple objective and constraints, where constructed portfolios are actively rebalanced based on simulated future market scenarios and interval type-2 fuzzy sets. Furthermore, Mehlawat and Gupta [17] address fuzzy chanceconstrained multi-objective portfolio optimization problem with a hybridization of fuzzy simulation and real-coded genetic algorithm, where famous financial criteria, i.e. return, risk and liquidity, are characterized by two measure representing short- and long-term variants.

Additionally, there are several works to focus on different important perspectives for portfolio investment. For instance, Jalota, Thakur, and Mittal [11] focus on automatic process of fitting parameters for several types of multi-objective credibilistic portfolio selection problems with L-R fuzzy numbers, which are solved by entropy-cross entropy algorithm. Differently, Chen and Huang [8] present a fuzzy portfolio optimization scheme for numerous equity mutual funds using a cluster analysis based on rates of return, standard deviation, turnover rate, and Treynor index. Elsewhere, $\mathrm{Li}$ and $\mathrm{Xu}$ [13] introduce the concepts of $\lambda$-mean variance efficient portfolios and frontiers in order to take into account investors' different forecasts about future returns of securities.

\section{Our scheme}

First of all, we consider stock trading under the following situation. Concretely, an investor executes the trading at each end of month under no-short-sale constraint so as to avoid the possible non-negligible costs for borrowing stocks. Further, we suppose that he/she is accessible to cash (or bank account) with zero interest rate.

The architecture of our framework is summarized in Figure 1. That is, our investment scheme is composed of two modules featuring fuzzy KBSs: The module I firstly calculates technical indicators with several parameter sets, and then generates various investment candidates with three types of fuzzy IF-THEN trading rule bases, each of which is designed to perform well in a specific market phase. On the other hand, the module II assesses historical records of these investment candidates from multilateral criteria, which are integrated through fuzzy inference to create a promising investment portfolio. 


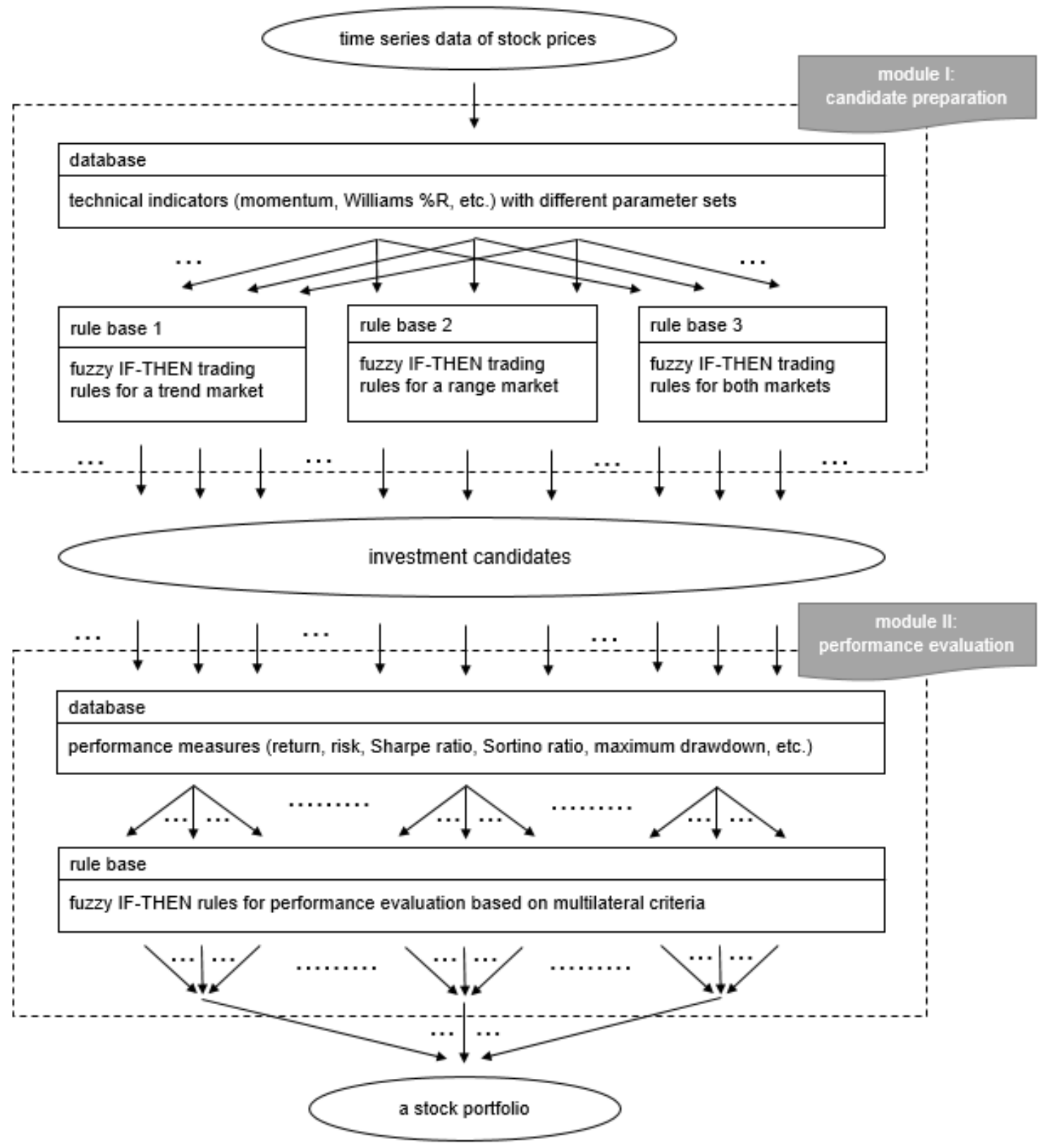

Fig. 1: Framework architecture

\subsection{Module I: Candidate preparation}

In this section, we describe fuzzy rule-based systems for technical trading to prepare investment candidates.

\subsubsection{Technical indicator}

We introduce the following three indicators, i.e. price momentum, moving standard deviation and Williams $\% R$, which are inputs of the fuzzy systems.

- Price momentum (PM) is the last closing price minus a closing price $n$ days ago.

- Moving standard deviation (MSD) is a standard deviation over recent $n$-days closing prices, which is a risk indicator. 
- Williams $\% R$ (WPR) is usually defined as (the last closing price - highest high)/(highest high - lowest low). Here, we use the highest/lowest closing price over the recent $n$ days instead of highest high and lowest low.

For the parameter $n$, we test three cases $n=20,40,60$ for each indicator. We note that "higher PM, lower MSD and higher WPR" yield a buy signal in the trend market, whereas "lower PM, higher MSD and lower WPR" do in the range market.

\subsubsection{Fuzzy system for technical trading}

Now, let us discuss fuzzy IF-THEN rules for technical trading. We adopt the following form of rules, the so-called zero-order Sugeno-Takagi-Kang model:

$$
\text { IF } x_{1, r} \text { is } A_{1, r} \text { AND } \cdots \text { AND } x_{I, r} \text { is } A_{I, r} \text {, THEN } y=b_{r} \in \mathbb{R}, r=1, \cdots R \text {, }
$$

where $R$ denotes the number of IF-THEN rules in a fuzzy system. Here, $x_{1, r}, \cdots, x_{I, r}$ in the premise parts stand for input variables, which are fuzzified by fuzzy sets $A_{1, r}, \cdots, A_{I, r}$. In this study, these inputs are the values of technical indicators for a stock, while the variable $y$ represents its rating.

Related to the determination of fuzzy IF-THEN trading rules, it is one of the most important issues for investors to properly judge whether the current market is in a trend or range phase. Depending on the judgement, investment decision for buying or selling a security may largely change even if the same technical indicators are used. However, it seems difficult to correctly detect the market phase in advance.

Then, we prepare two types of fuzzy rule bases: One makes profit in the trend market, and the other does in the range phase. Moreover, we also employ a rule base taking into account the both situations. Thus, we consider the three cases for trading rules, as shown in Table 1 .

Table 1: IF-THEN rules of fuzzy systems

\begin{tabular}{ccc|ccc}
\hline & IF & & \multicolumn{3}{c}{ THEN } \\
\hline PM & MSD & WPR & case I & case II & case III \\
\hline high & high & high & 0.5 & 0 & 0.25 \\
high & high & low & 0 & 0.5 & 0.25 \\
high & low & high & 1 & 0 & 0.5 \\
high & low & low & 0.5 & 0 & 0.25 \\
low & high & high & 0 & 0.5 & 0.25 \\
low & high & low & 0 & 1 & 0.5 \\
low & low & high & 0.5 & 0 & 0.25 \\
low & low & low & 0 & 0.5 & 0.25 \\
\hline
\end{tabular}

Table 1 shows the premise parts (IF parts) take the common form among the three fuzzy systems:

IF (PM is high/low) AND (MSD is high/low) AND (WPR is high/low), .

where "high" and "low" are fuzzy sets. On the other hand, for the consequent parts (THEN parts), there are three cases (i.e., case I, II and III), which are characterized by three rating value vectors, i.e., three patterns of $\left(b_{1}, \cdots, b_{8}\right)$ in Eq. (1), as shown in Table 1. Case I and II corresponds with a trading rule base for a trend and range market, respectively, whereas the investment strategy of case III takes into consideration the both market phases equally. 
For membership functions of the fuzzy sets "high" and "low", we use the following triangular ones, $M F_{\text {high }}$ and $M F_{\text {low }}$, respectively.

$$
\begin{aligned}
M F_{\text {high }}\left(x_{t, j, i}\right) & =\max \left\{\frac{x_{t, j, i}-a_{t, j, \min }}{a_{t, j, \max }-a_{t, j, \min }}, 0\right\}, \\
M F_{\text {low }}\left(x_{t, j, i}\right) & =\max \left\{-\frac{x_{t, j, i}-a_{t, j, \max }}{a_{t, j, \max }-a_{t, j, \min }}, 0\right\}, \\
a_{t, j, \max } & =\max _{i=1, \cdots, N}\left\{x_{t, j, i}\right\}, \\
a_{t, j, \min } & =\min _{i=1, \cdots, N}\left\{x_{t, j, i}\right\},
\end{aligned}
$$

where $x_{t, j, i}$ represents $j$-th technical indicator value $(j=1,2,3)$ for $i$-th stock $(i=1, \cdots, N)$ at time $t$. Here, we dynamically adjust the parameters $a_{t, j, \max }, a_{t, j, \min }$ because the market condition changes over time. In other words, since these technical indicator values $x_{t, j, i}$ may largely differ over time, it seems inappropriate to apply constant values to these parameters.

Lastly, crisp outputs $o_{t, i}(i=1, \cdots, N)$, i.e., resulting ratings of stocks, are obtained by the following defuzzification.

$$
\begin{aligned}
o_{t, i} & =\sum_{r=1, \cdots, 2^{3}} f_{r}\left(x_{t, i}\right) b_{r}, \\
f_{r}\left(x_{t, i}\right) & =\frac{M F_{r^{\prime}}\left(x_{t, 1, i}\right) M F_{r^{\prime \prime}}\left(x_{t, 2, i}\right) M F_{r^{\prime \prime \prime}}\left(x_{t, 3, i}\right)}{\sum_{r^{\prime}, r^{\prime \prime}, r^{\prime \prime \prime}=1,2}\left(M F_{r^{\prime}}\left(x_{t, 1, i}\right) M F_{r^{\prime \prime}}\left(x_{t, 2, i}\right) M F_{r^{\prime \prime \prime}}\left(x_{t, 3, i}\right)\right)}, \\
x_{t, i} & \equiv\left(x_{t, 1, i}, x_{t, 2, i}, x_{t, 3, i}\right), \quad r \equiv\left(r^{\prime}, r^{\prime \prime}, r^{\prime \prime \prime}\right) .
\end{aligned}
$$

Since 3 types of fuzzy systems with $3 \times 3 \times 3$ kinds of parameter sets are applied, we obtain 81 patterns of ratings $\left(o_{t, 1}, \cdots, o_{t, N}\right)$ for each time $t$. In this work, for each pattern, the investment proposal is to invest the highest rating stock. More strictly, a portfolio weight $\left(\omega_{t, i}\right)_{i=1, \cdots, N}$ is set to be $\omega_{t, i}=o_{t, i}\left(i=i_{\max }\right), \omega_{t, i}=0\left(i \neq i_{\max }\right)$, where a weight to cash is $1-\sum_{i=1}^{N} \omega_{t, i}$. Thus, fuzzy systems introduced in this section generate 81 patterns of investment proposals.

\subsection{Module II: Performance evaluation}

In this section, we design a fuzzy system to evaluate investment proposals generated by fuzzy IF-THEN trading rules introduced in Section 3.1. Particularly, the fuzzy system enables to integrate the practically well-known performance measures.

\subsubsection{Performance measure}

Suppose that there exist $N$ stocks and a risk-free asset (bank account or cash) with zero rate of return. Also we assume time indexes $t=0, \cdots, T$ denote trading dates, i.e., each end of month in the investment period. Then, given portfolio weight process $\left\{\left(\omega_{t, 1}, \cdots, \omega_{t, N+1}\right)\right\}_{t=0, \cdots, T-1}$, the portfolio value and return process, $\left\{V_{t}\right\}_{t=0, \cdots, T}$ and $\left\{R_{t}\right\}_{t=1, \cdots, T}$, are defined as follows.

$$
\begin{aligned}
V_{t+1} & =V_{t}\left(1+\sum_{i=1}^{N} \omega_{t, i} y_{t+1, i}\right)-\sum_{i=1}^{N} c_{i}\left|\omega_{t, i} V_{t}-\omega_{t-1, i} V_{t-1}\left(1+y_{t, i}\right)\right|, \quad V_{0}=1, \\
R_{t+1} & =V_{t+1} / V_{t}-1,
\end{aligned}
$$

where $c_{i}$ and $y_{t, i}$ denote a transaction spread and rate of return for $i$-th stock, respectively $(i=1, \cdots, N)$. Remark that $\omega_{t, i}$ stands for a portfolio weight of $i$-th security during $[t, t+1)$, and $\left(\omega_{t, 1}, \cdots, \omega_{t, N+1}\right)$ satisfy $\omega_{t, i} \geq 0$ and $\sum_{i=1}^{N+1} \omega_{t, i}=1$, where a weight of cash $\omega_{t, N+1}$ is set to be $1-\sum_{i=1}^{N} \omega_{t, i}$. 
The penalty term $\sum_{i=1}^{N} c_{i}\left|\omega_{t, i} V_{t}-\omega_{t-1, i} V_{t-1}\left(1+y_{t, i}\right)\right|$ of Eq. (4) is the total transaction cost arising from the portfolio re-balance at time $t$. Since $\omega_{t-1, i}$ and $\omega_{t, i}$ are portfolio weights of the $i$-th security during $[t-1, t)$ and $[t, t+1), \omega_{t-1, i} V_{t-1}\left(1+y_{t, i}\right)$ and $\omega_{t, i} V_{t}$ indicate the values of the $i$-th security before and after the position change at time $t$, respectively. That is, $\left|\omega_{t, i} V_{t}-\omega_{t-1, i} V_{t-1}\left(1+y_{t, i}\right)\right|$ represents the necessary amount of money for the position change of the $i$-th security at time $t$. Hence, the total transaction cost at time $t$ equals to the summation of $c_{i}\left|\omega_{t, i} V_{t}-\omega_{t-1, i} V_{t-1}\left(1+y_{t, i}\right)\right|$ for all $i=1, \cdots, N$. In this paper, we set $c_{i}=10$ bps for all stocks.

In the following, we briefly describe the well-known performance measures used in our fuzzy inference system.

- Compound Return (CR): We define CR as the annualized geometric average of the portfolio returns $\left\{R_{t}\right\}$ in Eq. (4), which is a standard measure of investment returns.

$$
C R \equiv\left\{\prod_{t=1}^{T}\left(1+R_{t}\right)\right\}^{12 / T}-1 .
$$

- Standard Deviation (SD), Downside Deviation (DD): SD is a well-known investment risk measure defined as the annualized standard deviation of $\left\{R_{t}\right\}$, while DD only regards negative returns as risk.

$$
\begin{aligned}
S D & \equiv\left\{\frac{12}{T} \sum_{t=1}^{T}\left(R_{t}-\bar{R}\right)^{2}\right\}^{1 / 2}, \bar{R} \equiv \frac{1}{T} \sum_{t=1}^{T} R_{t}, \\
D D & \equiv\left\{\frac{12}{T} \sum_{t=1}^{T} \min \left(0, R_{t}\right)^{2}\right\}^{1 / 2} .
\end{aligned}
$$

- Maximum Drawdown (MDD):

$$
M D D \equiv \max _{1 \leq t \leq T} \frac{M_{t}-V_{t}}{M_{t}}, \quad M_{t} \equiv \max _{0 \leq s \leq t} V_{s} .
$$

The drawdown is the decline from the past peak value $M_{t}$ to the present value $V_{t}$. In general, portfolio performance depends on the investment timing. The MDD contributes to the performance analysis because it is independent of the investment timing given the horizon $[0, T]$.

- Sharpe Ratio (ShR): ShR is usually defined as portfolio excess average returns divided by portfolio standard deviation. Since interest rates on cash are assumed to be zero, we define ShR as follows.

$$
S h R \equiv A R / S D, \quad A R \equiv 12 \bar{R} .
$$

Here, AR denotes the annualized arithmetic average of $\left\{R_{t}\right\}$, which corresponds to a simple return.

- Sortino Ratio (SoR): SoR does not regard the upside deviation as risk while ShR penalizes the both upside and downside deviations, which is often pointed out as a weakness of ShR.

$$
S o R \equiv A R / D D \text {. }
$$

- Sterling Ratio (StR): StR is a measure of risk-adjusted return that uses drawdown measures as denominator. We adopt the following definition:

$$
S t R \equiv A R / M D D \text {. }
$$




\subsubsection{Fuzzy system for multilateral performance evaluation}

Suppose that there are $L$ kinds of investment proposals, which are evaluated with $M$ kinds of performance measures. In the current work, $L=81$ (Section 3.1) and $M=7$ (Section 3.2.1). Our fuzzy system is a nonlinear mapping from $\mathbb{R}^{M \times L}$ into $\mathbb{R}^{L}$, that is, the inputs are $M$ well-known performance measures $\left\{x_{t, l, m}\right\}_{l=1, \cdots, L, m=1, \cdots, M}$ and the output is a new integrated performance measure $\left\{\hat{x}_{t, l}\right\}_{l=1, \cdots, L}$. More concretely, our fuzzy system implements the following procedures at each investment time $t=t_{s}, \cdots, t_{e}$, where $t_{s}>0, t_{e}=T$.

(i) Firstly, performance measures $\left\{x_{t, l, m}\right\}_{l=1, \cdots, L, m=1, \cdots, M}$ are calculated by time-series of the past portfolio values $\left\{V_{s, l} ; 0 \leq s \leq t\right\}$ for each portfolio $l=1, \cdots, L$ as the inputs.

(ii) Secondly, these inputs are fuzzified by the following triangular membership functions associated with three kinds of fuzzy sets $X_{k}, k=1,2,3$, i.e. High, Medium and Low.

$$
\begin{aligned}
M F_{1}\left(x_{t, l, m}\right) & =\max \left\{\frac{x_{t, l, m}-a_{t, m, 2}}{a_{t, m, 1}-a_{t, m, 2}}, 0\right\}, \\
M F_{2}\left(x_{t, l, m}\right) & =\min \left\{\frac{x_{t, l, m}-a_{t, m, 2}}{a_{t, m, 1}-a_{t, m, 2}},-\frac{x_{t, l, m}-a_{t, m, 2}}{a_{t, m, 2}-a_{t, m, 3}}\right\}+1, \\
M F_{3}\left(x_{t, l, m}\right) & =\max \left\{-\frac{x_{t, l, m}-a_{t, m, 2}}{a_{t, m, 2}-a_{t, m, 3}}, 0\right\}, \\
a_{t, m, 1} & =\max _{l=1, \cdots, L}\left\{x_{t, l, m}\right\}, \\
a_{t, m, 3} & =\min _{l=1, \cdots, L}\left\{x_{t, l, m}\right\}, \\
a_{t, m, 2} & =\left(a_{t, m, 1}+a_{t, m, 3}\right) / 2,
\end{aligned}
$$

for each $m=1, \cdots, M$ and $t=t_{s}, \cdots, t_{e}$.

(iii) Thirdly, we employ the following form of IF-THEN rule (e.g. [24,43]):

- The case of return or risk-adjusted return measures (CR, ShR, SoR, StR):

$*$ IF $x_{t, l, m}$ is $X_{1}$ (High), THEN $\hat{x}_{t, l, m, k}=1$.

$*$ IF $x_{t, l, m}$ is $X_{2}$ (Medium), THEN $\hat{x}_{t, l, m, k}=0.5$.

* IF $x_{t, l, m}$ is $X_{3}$ (Low), THEN $\hat{x}_{t, l, m, k}=0.25$.

- The case of risk measures (SD, DD, MDD):

* IF $x_{t, l, m}$ is $X_{1}$ (High), THEN $\hat{x}_{t, l, m, k}=0.25$.

$*$ IF $x_{t, l, m}$ is $X_{2}$ (Medium), THEN $\hat{x}_{t, l, m, k}=0.5$.

$*$ IF $x_{t, l, m}$ is $X_{3}$ (Low), THEN $\hat{x}_{t, l, m, k}=1$.

Here, the strength of each IF-THEN rule is evaluated by using grades of the membership function:

$$
g_{k}\left(x_{t, l, m}\right)=\frac{M F_{k}\left(x_{t, l, m}\right)}{\sum_{k=1,2,3, m=1, \cdots, M} M F_{k}\left(x_{t, l, m}\right)} .
$$

Note that we use $3 \times M$ number of IF-THEN rules.

(iv) Lastly, the output $\hat{x}_{t, l}$ is defuzzified as follows:

$$
\begin{aligned}
\hat{x}_{t, l} & =\sum_{k=1,2,3, m=1, \cdots, M} g_{k}\left(x_{t, l, m}\right) \hat{x}_{t, l, m, k} \\
& =\sum_{m=1, \cdots, M} \sum_{k=1,2,3} g_{k}\left(x_{t, l, m}\right) \hat{x}_{t, l, m, k} .
\end{aligned}
$$


Then, by comparing the values of this integrated measure $\left\{\hat{x}_{t, l}\right\}_{l=1, \cdots, L}$, it enables to obtain the proposed investment portfolio at time $t$. Specifically, we invest the top 25\% (20 patterns) investment candidates with proportional to the integrated measure values $\hat{x}_{t, l}$.

In addition, we explain the reason for the specification of our membership functions Eq. (11), whose typical example is described in Figure 2 for a performance measure CR. Namely, it is desirable to hold the following property for the membership functions: If a portfolio $l$ shows higher performance than a portfolio $l^{\prime}$ in terms of a measure $m$, the former is evaluated better than the latter in fuzzy logic-based evaluation, which is represented as follows.

- The case of return or risk-adjusted return measures (CR, ShR, SoR, StR):

$$
x_{t, l, m}>x_{t, l^{\prime}, m} \Rightarrow \sum_{k=1,2,3} g_{k}\left(x_{t, l, m}\right) \hat{x}_{t, l, m, k}>\sum_{k=1,2,3} g_{k}\left(x_{t, l^{\prime}, m}\right) \hat{x}_{t, l^{\prime}, m, k} .
$$

- The case of risk measures (SD, DD, MDD):

$$
x_{t, l, m}<x_{t, l^{\prime}, m} \Rightarrow \sum_{k=1,2,3} g_{k}\left(x_{t, l, m}\right) \hat{x}_{t, l, m, k}>\sum_{k=1,2,3} g_{k}\left(x_{t, l^{\prime}, m}\right) \hat{x}_{t, l^{\prime}, m, k} .
$$

One of the specification to preserve these relations is the three triangular membership functions with the parameters $a_{t, m, 1}, a_{t, m, 2}, a_{t, m, 3}$ in Eq. (11).

Again, we dynamically adjust the parameters $a_{t, m, 1}, a_{t, m, 2}, a_{t, m, 3}$ because the investment environment changes over time, which implies that it is not appropriate to assume these parameters to be constant.

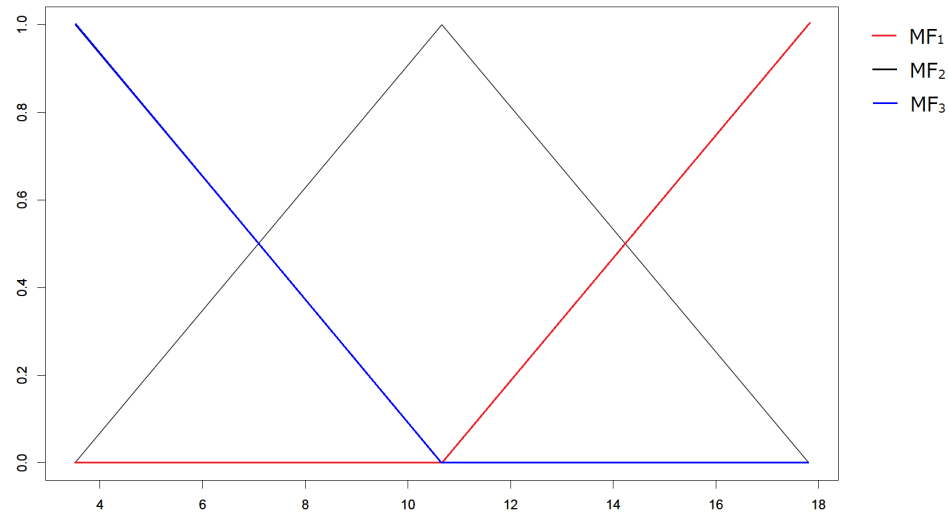

Fig. 2: Membership function (CR)

\section{Numerical experiment}

\subsection{Data}

We use daily Japanese stock closing price data from 2000/10/31 to $2017 / 4 / 28$, as listed in Table 2, where data source is Bloomberg. The 25 individual stocks in Table 2 are traded in First Section of Tokyo Stock Exchange. We select these securities in terms of liquidity, market capitalization and a length of periods when price data are accessible. In addition, Tokyo Stock Price Index (TOPIX) is also used as a trading instrument as well as a benchmark, that is 
market capitalization-weighted average of the securities traded in First Section of the Tokyo Stock Exchange. Note that there exist various Exchange Traded Funds (ETFs) for TOPIX.

As stated in Section 3.2.2, past performance evaluation is necessary for determination of the final investment proposal. Then, we set $t=0$ and $t=t_{s}$ to be $2000 / 10 / 31$ and $2002 / 9 / 30$, respectively.

Table 2: Dataset

\begin{tabular}{l|l}
\hline Bloomberg ticker code & Firm/index name \\
\hline TPX index & Tokyo Stock Price Index (TOPIX) \\
2914 JT Equity & Japan Tobacco Inc \\
4063 JT Equity & Shin-Etsu Chemical Co Ltd \\
4502 JT Equity & Takeda Pharmaceutical Co Ltd \\
4503 JT Equity & Astellas Pharma Inc \\
6501 JT Equity & Hitachi, Ltd. \\
6752 JT Equity & Panasonic Corporation \\
6758 JT Equity & Sony Corp \\
6861 JT Equity & KEYENCE CORPORATION \\
6902 JT Equity & Denso Corp \\
6954 JT Equity & Fanuc Corp \\
6981 JT Equity & Murata Manufacturing Co., Ltd. \\
7201 JT Equity & Nissan Motor Co Ltd \\
7203 JT Equity & Toyota Motor Corp \\
7267 JT Equity & Honda Motor Co Ltd \\
7751 JT Equity & Canon Inc \\
8031 JT Equity & Mitsui \& Co Ltd \\
8058 JT Equity & Mitsubishi Corp \\
8801 JT Equity & Mitsui Fudosan Co Ltd \\
8802 JT Equity & Mitsubishi Estate Co Ltd \\
9020 JT Equity & East Japan Railway Company \\
9022 JT Equity & Central Japan Railway Company \\
9432 JT Equity & Nippon Telegraph And Telephone Corp \\
9433 JT Equity & KDDI Corp \\
9437 JT Equity & NTT Docomo Inc \\
9984 JT Equity & SoftBank Group Corp \\
\hline
\end{tabular}

\subsection{Out-of-sample result}

Table 3 summarizes investment performances of 81 candidates and our proposed portfolios by calculating their quantiles ("0th", "25th", "50th", "75th", "100th") for each performance measure including a fuzzy-based integrated one ("Fuzzy criterion"). Especially for our scheme, in addition to the values of these performance measures, its ranking among $81+1$ patterns for each measure is presented.

It is observed that the investment performance drastically changes depending on the IFTHEN trading rules and the parameters of technical indicators, which causes substantial difficulty for selecting a portfolio in advance. Table 3 demonstrates that in this situation, our framework successfully constructs the well-performed portfolio from a number of candidates. For example, the risk-adjusted returns of our scheme are relatively high, which leads to the high ranking in terms of our fuzzy-based integrated measure. Moreover, as shown in Figure 3, our scheme substantially outperforms buy-and-hold of the market index (TOPIX), which has not performed well for a long time, under no-short-sale constraint. 
Table 3: Investment performance

\begin{tabular}{l|cccccccc}
\hline & $\mathrm{CR}(\%)$ & $\mathrm{SD}(\%)$ & $\mathrm{DD}(\%)$ & $\mathrm{MDD}(\%)$ & $\mathrm{ShR}(\%)$ & $\mathrm{SoR}(\%)$ & $\mathrm{StR}(\%)$ & Fuzzy criterion \\
\hline 0th & -5.66 & 31.69 & 18.51 & 85.01 & -9.62 & -14.89 & -3.04 & 0.28 \\
25 th & 2.38 & 27.05 & 16.65 & 74.79 & 22.41 & 37.76 & 8.29 & 0.36 \\
50 th & 7.86 & 20.50 & 10.87 & 42.48 & 57.07 & 104.15 & 25.07 & 0.59 \\
75 th & 13.30 & 14.53 & 8.12 & 33.04 & 74.76 & 133.49 & 39.71 & 0.69 \\
100th & 23.32 & 12.51 & 7.12 & 20.21 & 112.42 & 240.47 & 96.84 & 0.87 \\
\hline Our scheme & 14.78 & 15.32 & 7.84 & 27.68 & 97.99 & 191.42 & 54.23 & 0.81 \\
ranking of our scheme & 16 & 28 & 16 & 13 & 6 & 6 & 9 & 4 \\
\hline
\end{tabular}

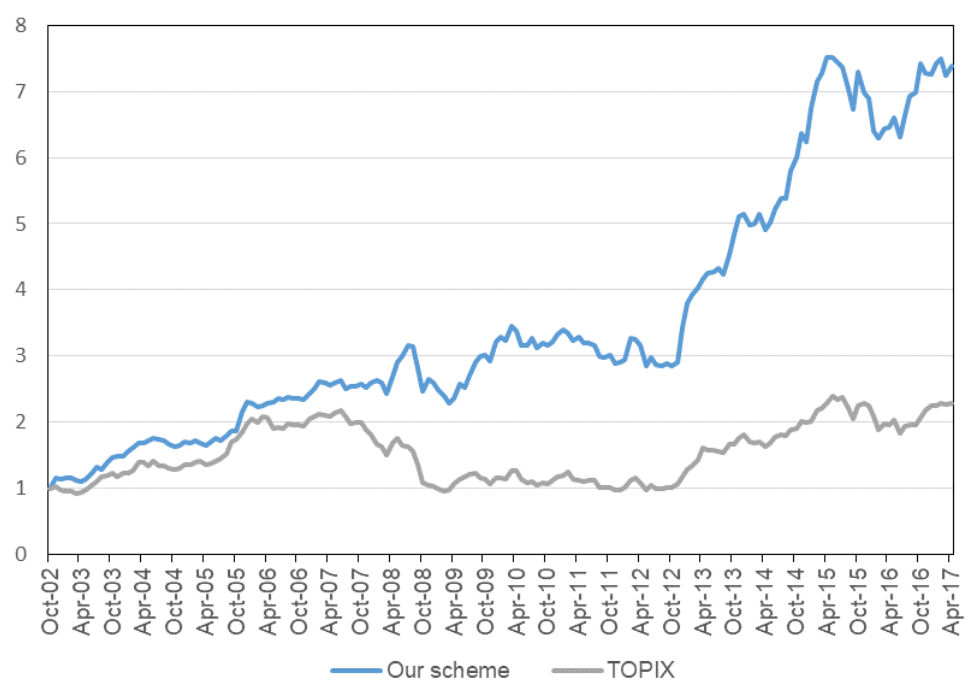

Fig. 3: Portfolio value

\section{Conclusion}

In this paper, we have developed a framework of technical trading by using fuzzy knowledgebased systems. Specifically, our framework consists of two module: In the 1st module, investment candidates are prepared based on three types of fuzzy IF-THEN trading rule bases that perform well in different market phases, where various parameter sets are also tested for technical indicators. Then, in the 2nd module, historical records of these candidates are evaluated through fuzzy inference integrating multiple performance criteria, which creates a series of portfolios with fine risk return profiles. Here, our trading scheme directly links to high performance because it generates a desirable portfolio in terms of multilateral measures frequently used in practice.

Further, an out-of-sample numerical experiment has confirmed that our framework successfully works under no-short-sale constraint in Japanese stock market, which has suffered from prolonged slump.

\section{Acknowledgement}

The authors are very grateful to Professor Hamido Fujita for his comments and suggestions. We are grateful to Takahiko Suenaga and Takeshi Hakamada at GCI Asset Management Inc. 
for valuable comments. This research is supported by CARF (Center for Advanced Research in Finance). Also, this work is supported by JSPS KAKENHI Grant Numbers JP17J09046 and JP17J09127.

\section{References}

[1] Cavalcante, R. C., Brasileiro, R. C., Souza, V. L., Nobrega, J. P., \& Oliveira, A. L., Computational intelligence and financial markets: A survey and future directions, Expert Systems with Applications 55 (2016), 194-211.

[2] Zadeh, L. A. Fuzzy sets. Information and control 8(3) (1965), 338-353.

[3] Markowitz, H. Portfolio selection. The Journal of Finance 7(1) (1952), 77-91.

[4] Michaud, R. O., The Markowitz optimization enigma: is' optimized'optimal?, Financial Analysts Journal 45(1) (1989), 31-42.

[5] Watada, J., Fuzzy portfolio selection and its applications to decision making, Tatra Mountains Mathematical Publication 13(4) (1997), 219-248.

[6] Ramaswamy, S., Portfolio selection using fuzzy decision theory, BIS Working Papers 59 (1998).

[7] Tanaka, H., Guo, P., \& Türksen, I. B., Portfolio selection based on fuzzy probabilities and possibility distributions, Fuzzy sets and systems 111(3) (2000), 387-397.

[8] Chen, L. H., \& Huang, L., Portfolio optimization of equity mutual funds with fuzzy return rates and risks. Expert Systems with Applications 36(2) (2009), 3720-3727.

[9] Fang, Y., Lai, K. K., \& Wang, S. Y., Portfolio rebalancing model with transaction costs based on fuzzy decision theory, European Journal of Operational Research 175(2) (2006), 879-893.

[10] Huang, X., Risk curve and fuzzy portfolio selection, Computers $\&$ Mathematics with Applications 55(6) (2008), 1102-1112.

[11] Jalota, H., Thakur, M., \& Mittal, G., Modelling and constructing membership function for uncertain portfolio parameters: A credibilistic framework, Expert Systems with Applications 71 (2017), 40-56.

[12] Li, X., Guo, S., \& Yu, L, Skewness of fuzzy numbers and its applications in portfolio selection, IEEE Transactions on Fuzzy Systems 23(6) (2015), 2135-2143.

[13] Li, J., \& Xu, J., A novel portfolio selection model in a hybrid uncertain environment, Omega 37(2) (2009), 439-449.

[14] Li, J., \& Xu, J., Multi-objective portfolio selection model with fuzzy random returns and a compromise approach-based genetic algorithm, Information Sciences 220 (2013), 507-521.

[15] Liu, S. T., A fuzzy modeling for fuzzy portfolio optimization, Expert Systems with Applications 38(11) (2011), 13803-13809.

[16] Mehlawat, M. K., Credibilistic mean-entropy models for multi-period portfolio selection with multi-choice aspiration levels. Information Sciences 345 (2016), 9-26. 
[17] Mehlawat, M. K., \& Gupta, P., Fuzzy chance-constrained multiobjective portfolio selection model, IEEE Transactions on Fuzzy Systems 22(3) (2014), 653-671.

[18] Nguyen, T. T., \& Gordon-Brown, L., Constrained fuzzy hierarchical analysis for portfolio selection under higher moments, IEEE Transactions on Fuzzy Systems 20(4) (2012), 666682 .

[19] Nguyen, T. T., Gordon-Brown, L., Khosravi, A., Creighton, D., \& Nahavandi, S., Fuzzy portfolio allocation models through a new risk measure and fuzzy Sharpe ratio, IEEE Transactions on Fuzzy Systems 23(3) (2015), 656-676.

[20] Pai, G. V., Fuzzy Decision Theory based Metaheuristic Portfolio Optimization and Active Rebalancing using Interval Type-2 Fuzzy Sets, IEEE Transactions on Fuzzy Systems 25(2) (2016), 377-391.

[21] Wang, B., Wang, S., \& Watada, J., Fuzzy-portfolio-selection models with value-at-risk, IEEE Transactions on Fuzzy Systems 19(4) (2011), 758-769.

[22] Zhou, J., Li, X., \& Pedrycz, W., Mean-Semi-Entropy Models of Fuzzy Portfolio Selection, IEEE Transactions on Fuzzy Systems 24(6) (2016), 1627-1636.

[23] Mamdani, E. H., \& Assilian, S., An experiment in linguistic synthesis with a fuzzy logic controller, International Journal of Man-Machine Studies 7(1) (1975), 1-13.

[24] Takagi, T., \& Sugeno, M., Fuzzy identification of systems and its applications to modeling and control, IEEE transactions on systems, man, and cybernetics 1 (1985), 116-132.

[25] Dourra, H., \& Siy, P., Investment using technical analysis and fuzzy logic. Fuzzy sets and systems 127(2) (2002), 221-240.

[26] Dymova, L., Sevastianov, P., \& Bartosiewicz, P. A new approach to the rule-base evidential reasoning: Stock trading expert system application. Expert Systems with Applications 37(8) (2010), 5564-5576.

[27] Kosaka, M., Mizuno, H., Sasaki, T., Someya, R., \& Hamada, N., Applications of fuzzy logic/neural network to securities trading decision support system, Systems, Man, and Cybernetics, 'Decision Aiding for Complex Systems, Conference Proceedings., 1991 IEEE International Conference on, 1913-1918.

[28] Lam, S. S. (2001). A genetic fuzzy expert system for stock market timing, Evolutionary Computation, 2001, Proceedings of the 2001 Congress on, Vol. 1, 410-417.

[29] Lincy, G. R. M., \& John, C. J., A multiple fuzzy inference systems framework for daily stock trading with application to NASDAQ stock exchange, Expert Systems with Applications: An International Journal 44(C) (2016), 13-21.

[30] Simutis, R., Fuzzy logic based stock trading system, Computational Intelligence for Financial Engineering (CIFEr), 2000. Proceedings of the IEEE/IAFE/INFORMS 2000 Conference on, 19-21.

[31] Yunusoglu, M. G., \& Selim, H., A fuzzy rule based expert system for stock evaluation and portfolio construction: An application to Istanbul Stock Exchange, Expert Systems with Applications 40(3) (2013) 908-920. 
[32] Chourmouziadis, K., \& Chatzoglou, P. D., An intelligent short term stock trading fuzzy system for assisting investors in portfolio management, Expert Systems with Applications 43 (2016), 298-311.

[33] Huang, C. J., Yang, D. X., \& Chuang, Y. T., Application of wrapper approach and composite classifier to the stock trend prediction, Expert Systems with Applications 34(4) (2008), $2870-2878$.

[34] Patel, J., Shah, S., Thakkar, P., \& Kotecha, K., Predicting stock market index using fusion of machine learning techniques, Expert Systems with Applications 42(4) (2015), 2162-2172.

[35] Patel, J., Shah, S., Thakkar, P., \& Kotecha, K., Predicting stock and stock price index movement using trend deterministic data preparation and machine learning techniques, Expert Systems with Applications 42(1) (2015) 259-268.

[36] Rather, A. M., Agarwal, A., \& Sastry, V. N., Recurrent neural network and a hybrid model for prediction of stock returns, Expert Systems with Applications 42(6) (2015), 3234-3241.

[37] Korol, T., A fuzzy logic model for forecasting exchange rates, Knowledge-Based Systems 67 (2014), 49-60.

[38] Cai, Q., Zhang, D., Zheng, W., \& Leung, S. C., A new fuzzy time series forecasting model combined with ant colony optimization and auto-regression, Knowledge-Based Systems $\mathbf{7 4}$ (2015), 61-68.

[39] Hadavandi, E., Shavandi, H., \& Ghanbari, A., Integration of genetic fuzzy systems and artificial neural networks for stock price forecasting, Knowledge-Based Systems 23(8) (2015), $800-808$.

[40] Singh, P., \& Borah, B., High-order fuzzy-neuro expert system for time series forecasting, Knowledge-Based Systems 46 (2013), 12-21.

[41] Bellman, R. E., \& Zadeh, L. A., Decision-making in a fuzzy environment, Management science 17(4) (1970), B-141.

[42] Zadeh, L. A., Fuzzy sets as a basis for a theory of possibility, Fuzzy sets and systems 1(1) (1978), 3-28.

[43] Sugeno, M., \& Kang, G. T., Structure identification of fuzzy model, Fuzzy sets and systems, 28(1) (1988), 15-33. 